\title{
tDCS confers neuroprotection by regulating LAT1-isoleucine-CBFB-PTEN signaling after rat cerebral ischemia-reperfusion injury
}

\author{
Xujin Yao ${ }^{1}$, Jinyang Ren ${ }^{1}$, Yu Cui ${ }^{1}$, Songfeng Chen ${ }^{2}$, Jing Cheng ${ }^{3}$, Jingchen Gao ${ }^{1}$, \\ Xiaohong Huang ${ }^{1}$, Jiangdong $\mathrm{Sun}^{1}$, Xiangyu Xu${ }^{1}$, Xiangyi Kong ${ }^{1}$, Wenjie Hu${ }^{1}$, Tao Lin ${ }^{1}$, \\ Fengyuan $\mathrm{Che}^{4}$, and Qi Wan ${ }^{1}$ \\ ${ }^{1}$ Qingdao University \\ ${ }^{2}$ Tsinghua University \\ ${ }^{3}$ Wuhan University \\ ${ }^{4}$ Linyi People's Hospital
}

October 26, 2021

\begin{abstract}
Background and Purpose: Isoleucine is a branched-chain amino acid serving as an essential nutrient resource and metabolic. However, its role in cerebral ischemic stroke remains unknown. Experimental Approach: Middle cerebral artery occlusion (MCAO) was used to mimic in vivo model of stroke. Oxygen-glucose deprivation insult (OGD) was used to injure cultured cortical neurons. High-Performance Liquid Chromatography (HPLC) was used to measure the level of isoleucine. A western blot assay and immunofluorescent staining were used to measure the level of CBFB and PTEN. TTC staining was used to measure the infarct size. Cell death and viability were assessed by LDH and CCK8 assays. DCS was used to stimulate cortical neurons. tDCS was used to stimulate the cortex. Key Results: Extraneuronal isoleucine is decreased and intraneuronal isoleucine is increased after rat cerebral I/R injury. Reducing intraneuronal isoleucine via inhibition of its transporter, LAT1 promotes neuronal survival whereas supplementing isoleucine aggravates neuronal damage. Isoleucine downregulates the expression of $\mathrm{CBFB}$, and that acts upstream of PTEN to mediate isoleucine-induced neuronal damage after OGD insult. To identify the therapeutic approach that suppresses the ischemia-induced increase of intraneuronal isoleucine, we tested the effect of tDCS on isoleucine. Our data suggest that Cathodal tDCS can reduce cerebral infarct size. And such neuroprotection is mediated through reducing LAT1-dependent increase of intraneuronal isoleucine. Conclusions and Implications: This study identifies LAT1- dependent increase of intraneuronal isoleucine promotes neuronal death after rat cerebral I/R injury. Our results indicate that tDCS protects against rat cerebral I/R injury through regulating LAT1-isoleucine-CBFB-PTEN signaling.
\end{abstract}

\section{INTRODUCTION}

Isoleucine is one of the three branched chain amino acids (BCAAs). Ingestion of BCAAs is effective for promoting rehabilitation of post-stroke patients and decreasing inflammation $(1,2)$. In the brain, isoleucine readily crosses the $\mathrm{BBB}$ and contributes to the production of neurotransmitters as nitrogen donors (3). Metabolite profiling has identified a decreased level of isoleucine in the plasma of acute stroke patients (4). Recent studies suggest that high concentration of isoleucine is associated with increased risk of cardiovascular diseases (5). However, the role of isoleucine in cerebral ischemic stroke is not clear.

CBFB (core binding factor $\beta$ ) plays a critical role in enhancing the DNA-binding activity and the stability of Runx protein (6). CBFB deficiency has been shown to result in embryo death (7). CBFB is also found 
to involve in hematopoiesis and bone development $(6,7)$. However, little is known about CBFB in cerebral ischemic stroke.

Transcranial direct-current stimulation (tDCS) is a promising non-invasive method to induce neuroplasticity within the cerebral cortex and augment stroke recovery $(8,9)$. However, the molecular mechanisms mediating the neuroprotective role of tDCS remains largely unknown. Direct-current electrical fields (DCSs) promote nerve growth and axon regeneration (10). Our previous study provides the first evidence that DCSs at physiological strengths guide the migration of neuronal stem/progenitor cells (NSPCs)(10), suggesting DCS as a potential approach in the treatment of neurological disorders.

In this study, we set out to elucidate the role of isoleucine in the injured neurons following cerebral ischemiareperfusion injury. We found unexpectedly that intraneuronal isoleucine is aggregated after cerebral ischemiareperfusion injury. Increased activity of LAT1 (an L-type amino acid transporter) leads to the elevation of intraneuronal isoleucine in cortical cultures subjected to oxygen-glucose deprivation (OGD). Reducing the level of intraneuronal isoleucine promotes cell survival after cerebral ischemia-reperfusion injury, and supplementing isoleucine aggravates the neuronal damage. We reveal that isoleucine acts upstream to reduce the expression of CBFB, and that the phosphatase PTEN acts downstream of CBFB to mediate isoleucineinduced neuronal damage after OGD insult. Interestingly, we demonstrate that DCS reduces the level of intraneuronal isoleucine in cortical cultures subjected to OGD, and that tDCS decreases the cerebral infarct volume of rat following middle cerebral artery occlusion (MCAO) through reducing LAT1-depencent increase of intraneuronal isoleucine. Together, these results lead us to conclude that LAT1 overactivationdependent isoleucine-CBFB-PTEN signal transduction pathway may mediate ischemic neuronal injury, and that tDCS exerts its neuroprotective effect by suppressing LAT1 overactivation-dependent signaling after cerebral ischemia-reperfusion injury.

\section{METHODS}

\section{Animals}

All animal experiments were approved and carried out in compliance with the IACUC guidelines of Qingdao University School of Medicine. All animal use and experimental protocols were approved and carried out in compliance with the IACUC guidelines and Animal Care and Ethics Committee of Qingdao University. All studies involving animals are reported in accordance with the ARRIVE guidelines for reporting experiments involving animals(11). Male Sprague-Dawley rats (250 - 300g, Qingdao Daren Fucheng Animal Husbandry Co. Ltd, China) were housed with $2-3$ rats per cage on a $12 \mathrm{~h}$ light/dark cycle in a temperature-controlled room $\left(23{ }^{\circ} \mathrm{C}-25{ }^{\circ} \mathrm{C}\right)$, with free access to food and water. Animals were allowed at least 3 days to acclimatize before experimentation. Samples were assigned to the experimental groups by random, for data collection and procession. Rats were placed in a plastic restraining holder, CSF ([?]50 $\mu \mathrm{L})$ was collected from the cisterna magna at $0 \mathrm{~h}, 3 \mathrm{~h}$ and $6 \mathrm{~h}$ after reperfusion, using a 27-gauge winged needle set attached to a $1 \mathrm{~cm}$ syringe. The experiments were performed by investigators blinded to the groups for which each animal was assigned.

\section{PTEN knockout mice}

Tamoxifen was used to induce the development of conditionally PTEN-knockout mice. First, we performed an indoor hybridization of the Ubc-CreER transgenic line of the Shanghai Model Biological Research Center with the PTEN LoxP/LoxP mice of Qingdao University. Genomic DNA was extracted from the tail tissues of F1 and F2 progeny, and then the genotype of each mouse (CreER transgene, focusing on PTEN) was studied. Finally, the genotypes were evaluated by PCR using PTEN LoxP and Cre as primers. PTEN LoxP forward CTCCTCTACTCCATTCTTCCC, and reverse, ACTCCCACCAATGAACAAAC; Cre forward, GCGGTCTGG CAGTAAAAACTATC, and reverse, GTGAAACAGCATTGCTGTCACTT. At the age of 4-6 weeks, the Pre-mutant mice (PTENLoxP/LoxP Ubc- CreER +) and control mice (PTEN LoxP/LoxP UbcCreER-) were given intraperitoneal infusion. Investigation of PTEN mouse phenotype was at the earliest 3 days after the ultimate infusion. The mice (tamoxifen-induced recombination) usually co-exist with different proportions of PTEN-wild-type cells and PTEN-knockout cells. PTEN was not removed from all cells. 


\section{Transient focal cerebral ischemia}

Transient focal cerebral ischemia was operated by suture occlusion technique as described in our previous study (12). The whole process is applied as our laboratory described. Male SD rats weighing 250- $300 \mathrm{~g}$ were anesthetized under gas condition of $4 \%$ isoflurane in $70 \% \mathrm{~N}_{2} \mathrm{O}$ and $30 \% \mathrm{O}_{2}$ and body temperature was remained during and after surgery. The middle incision was made on the neck, the right external carotid artery (ECA) was carefully exposed and dissected. A 3-0 monofilament nylon suture was inserted from the ECA into the right internal carotid artery to occlude the origin of the right middle cerebral artery (MCA) (approximately $22 \mathrm{~mm}$ ). After $60 \mathrm{~min}$ of occlusion, the suture was removed to allow reperfusion, the ECA was ligated, and the wound was closed. Sham-operated rats underwent identical surgery except that the suture was inserted and withdrawn immediately. Rectal temperature was maintained at $37.0 \pm 0.5^{\circ} \mathrm{C}$ using a heating pad and heating lamp. Brain samples were acquired after $24 \mathrm{~h}$ after middle cerebral artery occlusion (MCAO), before reperfusion with ice-cold $0.9 \%$ saline and decapitated, rats were anesthetized with $4 \%$ isoflurane in $70 \% \mathrm{~N}_{2}$ and $30 \% \mathrm{O}_{2}$. The peri-infarct tissues of ipsilateral hemispheres of the brains were rapidly removed for Western blotting, frozen sectioning and the sliced brain for TTC (2,3,5-triphenyltetrazolium chloride) staining. JPH203(10 $\mu \mathrm{M}, 1 \mu \mathrm{L}, \mathrm{S} 8667$, Selleckchem, USA) were i. c. v. injected prior to MCAO surgery. At $1 \mathrm{~h}$ after MCAO insult, isoleucine $(10 \mathrm{mg} / \mathrm{kg}$ ) (I2752, Sigma, USA) was intraperitoneally administrated.

\section{Infarct measurement}

TTC assay and infarct volume measurement were performed as described previously by us(12). Infarct volumes (in $\mathrm{mm}^{3}$ ) were derived after the animals were sacrificed $24 \mathrm{~h}$ after MCAO onset and the brains harvested and frozen to facilitate sectioning into $5 \times 2 \mathrm{~mm}$ coronal sections. Brain slices were stained for $30 \mathrm{~min}$ at 37 with $2 \%$ TTC (2,3,5-triphenyltetrazolium chloride: Sigma Aldrich, St. Louis, MD, USA) in phosphatebuffered saline (PBS) and the slices were fixed in $4 \%$ paraformaldehyde at 4 degC. The normal area of brain was stained red, whereas infarct area remained unstained. All images were collected and photographed with a scanner. The areas of the infarct, ipsilateral hemisphere and contralateral hemisphere were measured using NIH Image (Software version 1.52a) and volumes were derived by integrating the edema-corrected infarct areas (area of contralateral hemisphere minus area of healthy tissue in ipsilateral hemisphere).

\section{Primary cortical neuron culture}

The cortical neuronal cultures were prepared from rat at gestation day 17 as described by us previously (13). Briefly, dissociated neurons were suspended in plating medium (Neurobasal medium, 2\% B-27 supplement, $0.5 \%$ FBS, $0.5 \mu \mathrm{M}$ L-glutamine, and $25 \mu \mathrm{M}$ glutamic acid) and plated on poly-D-lysine coated Petri dishes. After 1.0 day in culture, half of the plating medium was removed and replaced with maintenance medium (Neurobasal medium, 2\% B-27 supplement, and $0.5 \mathrm{mM}$ L-glutamine). Thereafter, maintenance medium was changed in the same manner every 3.0 days. The cultured neurons were used for experiments at 12 days after plating.

\section{Oxygen-glucose deprivation insult}

The method of oxygen-glucose deprivation insult (OGD) was described in our previous study (13). To initiate the OGD challenge, cells were transferred to deoxygenated glucose-free extracellular solution (ECS) (in mM: $116 \mathrm{NaCl}, 5.4 \mathrm{KCl}, 0.8 \mathrm{MgSO}_{4}, 1.0 \mathrm{NaH}_{2} \mathrm{PO}_{4}, 1.8 \mathrm{CaCl}_{2}$, and $26 \mathrm{NaHCO}_{3}$ ), introduced into a specialized chamber, and maintained at $37^{\circ} \mathrm{C}$ in $95 \% \mathrm{~N}_{2}$ and $5 \% \mathrm{CO}_{2}$ for $2 \mathrm{~h}$. Neurons were removed from the chamber, transferred to maintenance medium, and returned to the incubator. For sham group treatment, cultures were transferred to the standard ECS (in mM: $116 \mathrm{NaCl}, 5.4 \mathrm{KCl}, 0.8 \mathrm{MgSO}_{4}, 1.0 \mathrm{NaH}_{2} \mathrm{PO}_{4}, 1.8 \mathrm{CaCl}_{2}, 26$ $\mathrm{NaHCO}_{3}$, and 33 glucose), introduced into the chamber maintained at $37^{\circ} \mathrm{C}$ in $95 \%$ air and $5 \% \mathrm{CO}_{2}$. After 1 $\mathrm{h}$ incubation, the neurons were transferred to maintenance medium and returned to the original incubator. JPH203(5 $\mu \mathrm{M}$ in 0.05\% DMSO, S8667, Selleckchem, USA) were applied prior to the OGD insult. At $1 \mathrm{~h}$ after reoxygenation and glucose deprivation, isoleucine $(50 \mu \mathrm{M})$ was administrated. Bisperoxovanadium (pyridine-2-carboxyl) [BpV(pic)] (Santa Cruz, CA) was applied to primary neuron 30min before OGD insult (14). 


\section{Western blotting analysis}

Western blotting was performed as previously described by us (15). A membrane was blocked with $5 \%$ (wt/vol) BSA or $5 \%$ (wt/vol) nonfat dry milk in TBST (TBS containing $0.1 \%$ Tween 20 ) at room temperature for $60 \mathrm{~min}$, incubated with appropriate primary antibodies at $4{ }^{\circ} \mathrm{C}$ overnight and then with HRP-conjugated secondary antibodies at room temperature for $60 \mathrm{~min}$. Antigen-antibody complexes were detected using chemiluminescence reagents (Millipore). Primary antibody CBFB (rabbit, 1:1000, DF3273, Affinity, China), PTEN (rabbit, 1:100, \#9188, Cell Signaling Technology, USA) and $\beta$-actin (rabbit, 1:2000, bs-0061R, Bioss, China).

\section{Immunofluorescent staining and confocal microscopy}

Immunofluorescence staining was performed as described previously by us (16). For doubleimmunofluorescence staining, primary cultured neurons cells were seeded in 24-well culture plates plated with cell-climbing slices. Neurons were fixed in $4 \%$ paraformaldehyde (PFA) for 20 min. Fixed cells were then incubated in blocking buffer ( $5 \%$ donkey serum, $0.3 \%$ Triton X-100 in PBS) for 2 hours. Incubation with primary antibodies were carried out in 4 , overnight. Phosphate Buffered Saline (PBS) instead of primary antibody for negative control overnight at 4 . Primary antibodies used are CBFB (anti-rabbit, 1:500, DF3273, Affinity, China), MAP2 (anti-mouse, 1:1000, Sigma-Aldrich, M4403) and NeuN (anti-mouse, 1:500, MAB377, Millipore Sigma, USA). After washing with PBS for three times, Primary neuron or sections were washed 3 times with 1X PBS, then incubated with the appropriate secondary antibodies in 1X Phosphate buffer TBS (1:500, anti-mouse 4408S; anti-rabbit 4412S Cell Signaling Technology, USA) for $2 \mathrm{~h}$ at room temperature and then were rinsed with PBS for 3 times. Finally, glass coverslips or sections were kept in an oven at $30-32 * \mathrm{C}$ for $10-15 \mathrm{~min}$ to dry and covered using Cover Glass (Fisher Scientific) with Gold antifade (Life Technologies, catalog number P36941). Confocal images were acquired using a fluorescence confocal microscope (C2 Si, Nikon, Tokyo, Japan). All digital images were processed using the same settings to improve the contrast.

\section{High performance liquid chromatography}

CSF sample $(50 \mu \mathrm{L})$ was pipetted into $2.0 \mathrm{~mL}$ micro-centrifuge tube and $5 \mu \mathrm{L}$ of DTT (for reduction of disulphide bonds and to release bound cysteine and homocysteine from proteins) was added to it (17). Then the solution was vortexed for $30 \mathrm{~s}$. After that an equal volume $(50 \mu \mathrm{L})$ of $10 \%$ sulfosalicylic acid was added to precipitate the proteins, followed by vortex mixing for $2 \mathrm{~min}$. Then the samples were centrifuged at $25,000 \mathrm{rpm}$ for $10 \mathrm{~min}$ at $4{ }^{\circ} \mathrm{C}$ yielding a clear and kept at $-80^{\circ} \mathrm{C}$. Sample was derivatized as described. The chromatographic system (1290 Infinity II, Agilent Technologies, Germany). Amino acids were detected based on the retention time established for the individual amino acid under defined experimental conditions. Calculation was based on the area under peak established for a given amino acid of known concentration.

\section{Lentivirus transfection}

The lentiviral-based PLKO.1 plasmid vector encoding two shRNA specific for CBFB shRNA were purchased from Genchem Biotechnology Company (Shanghai, China). The sequences of the two CBFB-specific shRNAs, CBFB- 1: TGAGATTAAGTACACGGGCTT, CBFB- 2: CCGCGAGTGTGAGATTAAGTA. The CBFB-recombinant lentivirus and PTEN-recombinant lentivirus were constructed by Genchem Biotechnology Company (Shanghai, China). The transfection was performed 3 days prior to OGD insult as described (18).

\section{DCS and tDCS application}

In OGD model, steady DCSs were applied to cultured neurons in the culture chambers using methods described previously by us (10). For the DCS stimulation, agar-salt bridges were used to connect silver/silver chloride electrodes in beakers of Steinberg's solution, to pools of excess culture medium at either side of the chamber. Field strengths were measured directly at the beginning and end of the observation period. Culture conditions in control were identical except no DCSs were applied. HEPES acid (20 mM) was added to the 
culture medium, with $\mathrm{pH}$ adjusted to 7.4. The cells were stimulated by DCS at the current strength of 250 $\mathrm{mV} / \mathrm{mm}$ for $20 \mathrm{~min}$ at $3 \mathrm{~h}$ after reoxygenation following OGD.

In MCAO model, tDCS was applied in rat without re-anesthesia by a constant current stimulator (Schneider Electronics, Gleichen, Germany) that was specifically designed for application of low-intensity currents in small mammals (19). Epicranial electrode implant was carried out in rat 7 days before the MCAO operation. One electrode was positioned on each side of the cranium in a symmetrical way and fixed with nontoxic glass ionomer cement. The electrode over the ischemic cortex was connected to the cathodal terminal and the other electrode was connected to anodal terminal. Prior to stimulation, epicranial implanted electrode was filled with saline solution. The contact area of the electrode toward the skull was $3.5 \mathrm{~mm}^{2}$. The tDCS was applied in rat at current intensity of $100 \mu \mathrm{A}$ with a current density of $2.86 \mathrm{~mA} / \mathrm{cm}^{2}$. Rat underwent tDCS at $3 \mathrm{~h}$ after I/R for $10 \mathrm{~min}$, followed by $3 \mathrm{~min}$ rest and then $10 \mathrm{~min}$ stimulation, for a total 8 times of $10 \mathrm{~min}$ stimulation. To avoid a stimulation break effect, the current strength was ramped for $10 \mathrm{~s}$. The sham rat underwent the same procedure of stimulated groups, but no current was applied.

\section{Analysis of lactate dehydrogenase release and cell viability}

Neuronal viability or injury was evaluated using Cell Counting Kit 8 (CCK8; Dojindo, CK04) or LDH Release Assay Kit (Biovision, K311-400). The two methods were performed under the manufacturer's instructions. For the CCK8 assay, $10 \mu \mathrm{L}$ CCK8 reagent was added to the culture medium $4 \mathrm{~h}$ before analysis and Optical density $(\mathrm{OD})_{450}$ values were measured using a microplate reader (Molecular Devices, SpectraMax ABS). For the LDH assay, $100 \%$ cell death was induced with $0.5 \%$ Triton X-100 in sister culture and $\mathrm{OD}_{490}$ values were measured. The relative assessments of neuronal injury or viability were normalized by comparison with $100 \%$ cell death (LDH release) or with control cell as 100\% cell survival (CCK8 assay).

\section{Statistics}

Student's $t$ test or ANOVA test was used where appropriate to examine the statistical significance of the differences between groups of data. All results are presented as mean \pm SEM. Significance was placed at $p$ $<0.05$.

\section{RESULTS}

\section{Isoleucine is decreased in cerebral spinal fluid after rat cerebral ischemia-reperfusion injury}

To study the role of isoleucine in cerebral ischemia-reperfusion injury, we first measured the level of isoleucine in rat cerebral spinal fluid (CSF) after middle cerebral artery occlusion (MCAO, an in vivo rat model of cerebral ischemia-reperfusion injury) at 0,3 and $6 \mathrm{~h}$ after ischemia-reperfusion. The samples were analyzed by High Performance Liquid Chromatography (HPLC). Our results show that the concentration of isoleucine in CSF is significantly decreased after cerebral ischemia-reperfusion injury (Fig. 1a). These data suggest that the level of extracellular isoleucine in the damaged brain is decreased after rat cerebral ischemic-reperfusion injury.

\section{The level of intraneuronal isoleucine is increased after oxygen-glucose deprivation}

To determine whether the level of intraneuronal isoleucine is also decreased after rat cerebral ischemicreperfusion injury, we performed HPLC to measure intracellular isoleucine in rat neuronal culture after oxygen-glucose deprivation (OGD). Surprisingly, we found that the level of intraneuronal isoleucine is increased after OGD insult (Fig. 1b).

Increased activity of LAT1 leads to the elevation of intraneuronal isoleucine after OGD insult

To understand how OGD induces the elevation of intraneuronal isoleucine, we examined the effect of L-type amino acid transporter 1 (LAT1) on the level of intraneuronal isoleucine since isoleucine is known to enter into cell via LAT1 $(20,21)$. HPLC analyses show that of JPH203, a potent LAT1 inhibitor (22-24), blocks OGD-induced increase of intraneuronal isoleucine (Fig. 1b), implying that an increased activity of LAT1 leads to the increase of intraneuronal isoleucine after OGD insult. 


\section{Reducing the level of intraneuronal isoleucine promotes cell survival after cerebral ischemia-}

reperfusion injury

To determine whether the increased intraneuronal isoleucine has impact on the neuronal survival after cerebral ischemia-reperfusion injury, we first test the effect of isoleucine treatment in both MCAO and OGD models. We show that supplementing isoleucine increases the infarct volume in MCAO rats and also causes more severe damage in cortical neuronal cultures after OGD (Fig. 2a-c). We then tested the effect of LAT1 inhibitor JPH203 in rat MCAO model. We found that injection of JPH203 decreases the infarct volume in MCAO rats (Fig. 2d). In cultured cortical neurons, JPH203 treatment reduces the neuronal death after OGD insult (Fig. 2e-f). Together, these results indicate that the elevation of intraneuronal isoleucine by the increased activity of LAT1 promotes neuronal death and that reducing the level of intraneuronal isoleucine by LAT1 inhibitor protects against neuronal damage after cerebral ischemia-reperfusion injury.

Increase of intraneuronal isoleucine promotes neuronal death through CBFB after OGD insult

To understand the molecular mechanisms by which the increase of intraneuronal isoleucine promotes ischemic neuronal death, we examined the role of $\mathrm{CBFB}$, a co-binding factor of Runx protein in ischemia injury. Our results show that $\mathrm{CBFB}$ is expressed in rat brain, and that the expression of $\mathrm{CBFB}$ is decreased after ischemic stroke in the penumbra brain tissue of cerebral cortex and in primary neuron culture after OGD insult (Supplementary Fig. 1a; Fig. 3a-c; Supplementary Fig. 1b-c). We further show that CBFB knockdown by lentiviral CBFB shRNA treatment reduces neuronal viability in OGD-insulted cortical cultures, and that CBFB overexpression promotes neuronal survival (Supplementary Fig. 2a-d).

Based on the TRANSFAC Predicted Transcription Factor Targets dataset (https://genexplain.com/transfac/), we predicted that there might be interaction between Mammalian Target of Rapamycin pathway (mTOR) and CBFB (25). Since isoleucine is a regulator of mTOR signaling (26-28), we reason that isoleucine may be the upstream signaling of CBFB. As expected, we found that intraperitoneal injection of isoleucine in rat leads to a decreased level of CBFB in brain cortex tissue (Fig. 4a, Supplementary Fig. 3 a). In consistent with this observation, isoleucine treatment reduces the level of CBFB in neuronal culture (Fig. 4b, Supplementary Fig. 3b). Thus, intraneuronal isoleucine acts an upstream signal to downregulate CBFB in neurons.

The observed downregulation of CBFB by isoleucine leads us to propose that intraneuronal isoleucine may acts through CBFB to promote neuronal death after OGD insult. CBFB was overexpressed in cultured neurons before subjected to OGD insult. We found that overexpressing CBFB blocks isoleucine-induced enhancement of neuronal damage after OGD insult while knockdown of CBFB further increases isoleucineinduced neuronal damage (Fig. 4c-f). These data suggest that CBFB mediates isoleucine-induced neuronal damage.

\section{PTEN acts downstream of CBFB to mediate isoleucine increase-induced neuronal death after} OGD insult

PTEN is a key regulator in cell survival (29-31). PTEN-PI3K-AKT signaling is mediated by Runx $(32,33)$. Our data show that PTEN is decreased after overexpression of CBFB (Fig. 5a). On the contrast, knockdown of CBFB leads to the upregulation of PTEN (Fig. 5b). However, overexpression of PTEN does not alter the level of CBFB (Fig. 5c). Furthermore, there is no detected change of CBFB expression in cerebral cortex of Pten $^{-/-}$mouse (Fig. 5d, Supplementary Fig. 1d). These data suggest that PTEN is the downstream signal of CBFB in neurons.

We further show that there is an upregulation of PTEN in primary cortical neuron after OGD insult (Fig. 5e). Overexpression of CBFB reduces PTEN expression, whereas knockdown of CBFB upregulates PTEN expression in OGD-insulted neurons (Fig. 5f). These results support the notion that downregulation of CBFB leads to the upregulation of PTEN after OGD insult.

To determine whether PTEN acts downstream of CBFB to mediate isoleucine increase-induced neuronal death after OGD insult, we first show that isoleucine causes downregulation of CBFB but upregulation 
of PTEN (Fig. 6a). Then, by performing cell viability assay and LDH release assay we demonstrate that treatment of PTEN inhibitor bpV(pic) blocks isoleucine supplement-induced neuronal death after OGD insult (Fig. 6b-c). Taken together, we conclude that CBFB-PTEN signal pathway may mediate isoleucine increase-induced neuronal death after OGD insult.

\section{Application of tDCS alleviates ischemic neuronal injury through the reduction of intraneuronal isoleucine via inhibiting LAT1}

Our data thus far indicate that overactivation of LAT1 promotes ischemic neuronal damage through isoleucine-CBFB-PTEN signaling pathway after rat cerebral ischemia-reperfusion injury. As cathodal tDCS is shown to protect against cerebral ischemia injury $(8,9)$, we set up to determine whether LAT1-dependent signaling mediates cathodal tDCS-induced neuroprotective effect. We first confirmed that cathodal tDCS exerts neuroprotective effect in rat MCAO model (Fig. 7a) We then show that DCS results in a decreased level of intraneuronal isoleucine in the neuronal cultures (Fig. 7b). LAT1 inhibitor decreases intraneuronal isoleucine but does not further decrease the level of intraneuronal isoleucine (Fig. 7b), suggesting that DCS decreases intraneuronal isoleucine via inhibition of LAT1 in ischemic neurons. Finally, in OGD and MCAO models, we demonstrate that isoleucine supplement blocks DCS- and tDCS-induced neuroprotective effects, whereas the treatment of LAT1 inhibitor JPH203 does not further decrease ischemic neuronal injury after DCS or tDCS application (Fig. 7). Collectively, we conclude that tDCS decreases ischemic neuronal damage through the reduction of intraneuronal isoleucine via inhibiting LAT1.

\section{DISCUSSION}

Isoleucine is used as nutrition source in clinical vignettes (34-36). In the brain, isoleucine serves as important metabolic precursors, which are required for the synthesis of proteins and neurotransmitters, including dopamine, serotonin and norepinephrine (37). Studies have shown that acute supplementation of isoleucine reduces seizure propagation for patients with epilepsy (38). However, the role of isoleucine in cerebral ischemic stroke is not clear. The present study reveals a non-canonical role of isoleucine in rat model of cerebral ischemia-reperfusion injury.

We found that isoleucine is decreased in CSF in rats after cerebral ischemic reperfusion injury, which is consistent with the recent metabolomics profiling on plasma of the acute stroke patients (39-42). Unexpectedly, we further show that intraneuronal isoleucine is increased after OGD insult. Reducing intraneuronal isoleucine ameliorates the neuronal death, whereas injection of isoleucine aggravates the neuronal damage. Thus, aggregation of intraneuronal isoleucine is a potential target for the development of new ischemic stroke therapy.

CBFB is a non-DNA-binding partner of Runt-related transcription factors1-3 (Runx1-3)(43). CBFB forms a heterodimer with Runx, which enhances the transcription ability of Runx protein(44, 45). Although CBFB is expressed in the brain, little is known about the functional role of CBFB in the central nervous system. We found for the first time that CBFB is decreased in ischemic neurons, which contributes to the neuronal death after ischemia-reperfusion injury. Moreover, we demonstrate that the increase of isoleucine leads to the reduced level of CBFB in ischemic neurons, whereas PTEN acts downstream of CBFB to mediate isoleucine increase-induced neuronal death after OGD insult.

As a noninvasive brain stimulation, tDCS has been shown to exert neuroprotective effect in cerebral ischemiareperfusion injury by inhibition of neuroinflammation. DCS protects against ischemic neuronal death by reducing the activity of LAT1 and subsequent reduction of intraneuronal isoleucine. Thus, this study reveals a molecular mechanism by which tDCS confers neuroprotection through regulating LAT1-isoleucine-dependent signaling after cerebral ischemia-reperfusion injury. These findings support tDCS as a potential neuroprotection therapy for stroke patients.

Our data indicate that isoleucine supplement promotes neuronal damage after cerebral ischemia-reperfusion injury, which does not support the application that isoleucine supplement in ischemic stroke therapy. Recent studies seem to suggest the non-beneficial roles of isoleucine in several pathological conditions. Ingestion 
of BCAAs significantly promotes human platelet activity and arterial thrombosis formation in mice (46). Long-term application of BCAAs could lead to decreased tryptophan and reduced neuronal serotonin (47). Chronic high level of plasma isoleucine is shown to be positively associated with AD (48, 49). Thus, in-depth study is required to further uncover the role of isoleucine in pathological conditions.

This study has several limitations. First, we only show the increased import of isoleucine, which results in the aggregation of intraneuronal isoleucine. Further study needs to be done to determine how LAT1 is activated in ischemic conditions. Second, the dysfunction of isoleucine catabolism is likely to contribute to ischemia-induced reduction of isoleucine (50). Future studies will be performed to test this possibility. Third, although this study indicates that isoleucine results in downregulation of CBFB, more detailed studies are needed to establish how isoleucine downregulates CBFB. In addition, due to the unavailability of antiisoleucine antibody, we were unable to label and measure the reduction of intraneuronal isoleucine in the ischemic brain tissue in the rat MCAO model.

In conclusion, our study demonstrates that ischemia-induced increase of intraneuronal isoleucine promotes ischemic neuronal death. We provide evidence that tDCS treatment protects against ischemic neuronal death through regulating LAT1-isoleucine-CBFB-PTEN signaling after rat cerebral ischemia-reperfusion injury.

\section{DECLARATION OF TRANSPARENCY AND SCIENTIFIC RIGOUR}

This Declaration acknowledges that this paper adheres to the principles for transparent reporting and scientific rigour of preclinical research as stated in the BJP guidelines for Design \& Analysis, Immunoblotting and Immunochemistry, and Animal Experimentation, and as recommended by funding agencies, publishers and other organizations engaged with supporting research.

\section{REFERENCE}

1. Cole JT, Mitala CM, Kundu S, Verma A, Elkind JA, Nissim I, et al. Dietary branched chain amino acids ameliorate injury-induced cognitive impairment. Proc Natl Acad Sci U S A. 2010;107(1):366-71.

2. Cao Y, Liu K, Liu S, Guo L, Cai C, Yao JJBri. Isoleucine Regulates the Synthesis of Pancreatic Enzymes via the Activation of mRNA Expression and Phosphorylation in the Mammalian Target of Rapamycin Signalling Pathways in Pancreatic Tissues. Br J Pharmacol. 2019;2019:6302950.

3. Hutson S, Sweatt A, Lanoue KJTJon. Branched-chain [corrected] amino acid metabolism: implications for establishing safe intakes. J Nutr. 2005;135:1557S-64S.

4. Wang D, Kong J, Wu J, Wang X, Lai MJNl. GC-MS-based metabolomics identifies an amino acid signature of acute ischemic stroke. Neurosci Letters. 2017;642:7-13.

5. Ruiz-Canela M, Toledo E, Clish CB, Hruby A, Liang L, Salas-Salvado J, et al. Plasma Branched-Chain Amino Acids and Incident Cardiovascular Disease in the PREDIMED Trial. Clin Chem. 2016;62(4):582-92.

6. Qin X, Jiang Q, Matsuo Y, Kawane T, Komori H, Moriishi T, et al. Cbfb regulates bone development by stabilizing Runx family proteins. J Bone Miner Res. 2015;30(4):706-14.

7. Wang CQ, Chin DW, Chooi JY, Chng WJ, Taniuchi I, Tergaonkar V, et al. Cbfb deficiency results in differentiation blocks and stem/progenitor cell expansion in hematopoiesis. Leukemia. 2015;29(3):753-7.

8. Zhang K, Rui G, Zhang J, Guo L, An G, Lin J, et al. Cathodal tDCS exerts neuroprotective effect in rat brain after acute ischemic stroke. BMC Neuroscience . 2020;21(1):21.

9. Peruzzotti-Jametti L, Cambiaghi M, Bacigaluppi M, Gallizioli M, Gaude E, Mari S, et al. Safety and efficacy of transcranial direct current stimulation in acute experimental ischemic stroke. Stroke. 2013;44(11):316674 .

10. Li L, El-Hayek Y, Liu B, Chen Y, Gomez E, Wu X, et al. Direct-current electrical field guides neuronal stem/progenitor cell migration. Stem Cells. 2008;26(8):2193-200. 
11. Curtis M, Alexander S, Cirino G, Docherty J, George C, Giembycz M, et al. Experimental design and analysis and their reporting II: updated and simplified guidance for authors and peer reviewers. British Journal of Pharmacology. 2018;175(7):987-93.

12. Zhang Z, Chen J, Han X, Zhang Y, Liao H, Lei R, et al. Bisperoxovandium (pyridin-2-squaramide) targets both PTEN and ERK1/2 to confer neuroprotection. Bisperoxovandium (pyridin-2-squaramide) targets both PTEN and ERK1/2 to confer neuroprotection. British Journal of Pharmacology. 2017;174(8):641-56.

13. Liu B, Liao M, Mielke J, Ning K, Chen Y, Li L, et al. Ischemic insults direct glutamate receptor subunit 2-lacking AMPA receptors to synaptic sites. 2006;26(20):5309-19.

14. Liu R, Tang J, Pan M, Zhuang Y, Zhang Y, Liao H, et al. ERK 1/2 Activation Mediates the Neuroprotective Effect of $\mathrm{BpV}$ (pic) in Focal Cerebral Ischemia-Reperfusion Injury. 2018;43(7):1424-38.

15. Ning K, Pei L, Liao M, Liu B, Zhang Y, Jiang W, et al. Dual neuroprotective signaling mediated by downregulating two distinct phosphatase activities of PTEN. The Journal of Neuroscience. 2004;24(16):405260 .

16. Ning K, Li L, Liao M, Liu B, Mielke J, Chen Y, et al. Circadian regulation of GABAA receptor function by CKI epsilon-CKI delta in the rat suprachiasmatic nuclei. Nature Neuroscience. 2004;7(5):489-90.

17. Sharma G, Attri SV, Behra B, Bhisikar S, Kumar P, Tageja M, et al. Analysis of 26 amino acids in human plasma by HPLC using AQC as derivatizing agent and its application in metabolic laboratory. Amino Acids. 2014;46(5):1253-63.

18. Gao C, Wang X, Lu J, Li Z, Jia H, Chen M, et al. Mesenchymal stem cells transfected with sFgl2 inhibit the acute rejection of heart transplantation in mice by regulating macrophage activation. Stem Cell Res Ther. 2020;11(1):241.

19. Liebetanz D, Koch R, Mayenfels S, König F, Paulus W, Nitsche MJCnojotIFoCN. Safety limits of cathodal transcranial direct current stimulation in rats. Clinical Neuroscience. 2009;120(6):1161-7.

20. Li Y, Gao L, Luo X, Wang L, Gao X, Wang W, et al. Epigenetic silencing of microRNA-193a contributes to leukemogenesis in $\mathrm{t}(8 ; 21)$ acute myeloid leukemia by activating the PTEN/PI3K signal pathway. Blood. 2013;121(3):499-509.

21. Sano A, Shi H, Suzuki R, Shirataki Y, Sakagami H. Change in Amino Acid Pools During Neuronal Differentiation of PC12 Cells. In Vivo. 2018;32(6):1403-8.

22. Johansen ML, Bak LK, Schousboe A, Iversen P, Sorensen M, Keiding S, et al. The metabolic role of isoleucine in detoxification of ammonia in cultured mouse neurons and astrocytes. Neurochem Int. 2007;50(78):1042-51.

23. Okunushi K, Furihata T, Morio H, Muto Y, Higuchi K, Kaneko M, et al. JPH203, a newly developed anti-cancer drug, shows a preincubation inhibitory effect on L-type amino acid transporter 1 function. J Pharmacol Sci. 2020;144(1):16-22.

24. Patel M, Dalvi P, Gokulgandhi M, Kesh S, Kohli T, Pal D, et al. Functional characterization and molecular expression of large neutral amino acid transporter (LAT1) in human prostate cancer cells. Int J Pharm. 2013;443(1-2):245-53.

25. Tandon M, Chen Z, Pratap JJBcrB. Runx2 activates PI3K/Akt signaling via mTORC2 regulation in invasive breast cancer cells. Breast Cancer Research. 2014;16(1):R16.

26. Ye J, Palm W, Peng M, King B, Lindsten T, Li MO, et al. GCN2 sustains mTORC1 suppression upon amino acid deprivation by inducing Sestrin2. Genes Dev. 2015;29(22):2331-6.

27. Li H, Ye D, Xie W, Hua F, Yang Y, Wu J, et al. Defect of branched-chain amino acid metabolism promotes the development of Alzheimer's disease by targeting the mTOR signaling. Bioscience Reports. 
$2018 ; 38(4)$.

28. Tenno M, Shiroguchi K, Muroi S, Kawakami E, Koseki K, Kryukov K, et al. Cbfß2 deficiency preserves Langerhans cell precursors by lack of selective TGF $\beta$ receptor signaling. The Journal of Experimental Medicine. 2017;214(10):2933-46.

29. Zhao D, Qin XP, Chen SF, Liao XY, Cheng J, Liu R, et al. PTEN Inhibition Protects Against Experimental Intracerebral Hemorrhage-Induced Brain Injury Through PTEN/E2F1/beta-Catenin Pathway. Front Mol Neurosci. 2019;12:281.

30. Kath C, Goni-Oliver P, Müller R, Schultz C, Haucke V, Eickholt B, et al. PTEN suppresses axon outgrowth by down-regulating the level of detyrosinated microtubules. PLoS One. 2018;13(4):e0193257.

31. Sarn N, Jaini R, Thacker S, Lee H, Dutta R, Eng CJMp. Cytoplasmic-predominant Pten increases microglial activation and synaptic pruning in a murine model with autism-like phenotype. Molecular Psychiatry. 2020 .

32. Lim M, Zhong C, Yang S, Bell AM, Cohen MB, Roy-Burman P. Runx2 regulates survivin expression in prostate cancer cells. Lab Invest. 2010;90(2):222-33.

33. Farina N, Zingiryan A, Akech J, Callahan C, Lu H, Stein J, et al. A microRNA/Runx1/Runx2 network regulates prostate tumor progression from onset to adenocarcinoma in TRAMP mice. Oncotarget . 2016;7(43):70462-74.

34. Mori T, Yoshioka K. Quick and effective improvement of leucine enriched dietary supplement on malnutrition in acute stroke patients receiving enteral tube feeding. BMC Emerg Med. 2020;20(1):56.

35. De Simone R, Vissicchio F, Mingarelli C, De Nuccio C, Visentin S, Ajmone-Cat MA, et al. Branched-chain amino acids influence the immune properties of microglial cells and their responsiveness to pro-inflammatory signals. Biochim Biophys Acta. 2013;1832(5):650-9.

36. D’Antona G, Ragni M, Cardile A, Tedesco L, Dossena M, Bruttini F, et al. Branched-chain amino acid supplementation promotes survival and supports cardiac and skeletal muscle mitochondrial biogenesis in middle-aged mice. Cell Metab. 2010;12(4):362-72.

37. Holecek M. Branched-chain amino acids in health and disease: metabolism, alterations in blood plasma, and as supplements. Nutr Metab (Lond). 2018;15:33.

38. Gruenbaum SE, Dhaher R, Rapuano A, Zaveri HP, Tang A, de Lanerolle N, et al. Effects of BranchedChain Amino Acid Supplementation on Spontaneous Seizures and Neuronal Viability in a Model of Mesial Temporal Lobe Epilepsy. J Neurosurg Anesthesiol. 2019;31(2):247-56.

39. Szpetnar M, Matras P, Boguszewska-Czubara A, Kielczykowska M, Rudzki S, Musik I. Is additional enrichment of diet in branched-chain amino acids or glutamine beneficial for patients receiving total parenteral nutrition after gastrointestinal cancer surgery? Adv Clin Exp Med. 2014;23(3):423-31.

40. Holecek M. Branched-Chain Amino Acids and Branched-Chain Keto Acids in Hyperammonemic States: Metabolism and as Supplements. Metabolites. 2020;10(8).

41. Wang D, Kong J, Wu J, Wang X, Lai M. GC-MS-based metabolomics identifies an amino acid signature of acute ischemic stroke. Neurosci Lett. 2017;642:7-13.

42. Ruiz-Canela M, Guasch-Ferré M, Toledo E, Clish C, Razquin C, Liang L, et al. Plasma branched chain/aromatic amino acids, enriched Mediterranean diet and risk of type 2 diabetes: case-cohort study within the PREDIMED Trial. Diabetologia. 2018;61(7):1560-71.

43. Qin X, Jiang Q, Matsuo Y, Kawane T, Komori H, Moriishi T, et al. Cbfb regulates bone development by stabilizing Runx family proteins. J Bone Miner Res. 2015;30(4):706-14. 
44. Wang C, Chin D, Chooi J, Chng W, Taniuchi I, Tergaonkar V, et al. Cbfb deficiency results in differentiation blocks and stem/progenitor cell expansion in hematopoiesis. Leukemia. 2015;29(3):753-7.

45. Davis J, Rogers D, Adams L, Yong T, Jung J, Cheng B, et al. Association of core-binding factor $\beta$ with the malignant phenotype of prostate and ovarian cancer cells. Journal of Cellular Physiology. 2010;225(3):875-87.

46. Xu Y, Jiang H, Li L, Chen F, Liu Y, Zhou M, et al. Branched-Chain Amino Acid Catabolism Promotes Thrombosis Risk by Enhancing Tropomodulin-3 Propionylation in Platelets. Circulation. 2020;142(1):49-64.

47. Choi S, Disilvio B, Fernstrom MH, Fernstrom JD. Oral branched-chain amino acid supplements that reduce brain serotonin during exercise in rats also lower brain catecholamines. Amino Acids. 2013;45(5):113342.

48. Li H, Ye D, Xie W, Hua F, Yang Y, Wu J, et al. Defect of branched-chain amino acid metabolism promotes the development of Alzheimer's disease by targeting the mTOR signaling. Biosci Rep. 2018;38(4).

49. Griffin JW, Bradshaw PC. Amino Acid Catabolism in Alzheimer's Disease Brain: Friend or Foe? Oxid Med Cell Longev. 2017;2017:5472792.

50. Cole JT, Sweatt AJ, Hutson SM. Expression of mitochondrial branched-chain aminotransferase and alpha-keto-acid dehydrogenase in rat brain: implications for neurotransmitter metabolism. Front Neuroanat. 2012;6:18.

tDCS confers neuroprotection by regulating LAT1-isoleucine-CBFB-PTEN signaling after rat cerebral ischemia-reperfusion injury

Running title: Regulation of LAT1-isoleucine-CBFB-PTEN signaling by tDCS

Xujin $\mathrm{Yao}^{\mathrm{a}}$, Jinyang Ren ${ }^{\mathrm{a}}$, Yu Cui ${ }^{\mathrm{a}}$, Songfeng Chen ${ }^{\mathrm{b}}$, Jing Cheng ${ }^{\mathrm{b}}$, Xiaohong Huang ${ }^{\mathrm{a}}$, Jingchen $\mathrm{Gao}^{\mathrm{a}}$, Jiangdong Sun ${ }^{\mathrm{a}}$, Xiangyu $\mathrm{Xu}^{\mathrm{c}}$, Xiangyi Kong ${ }^{\mathrm{a}}$, Wenjie $\mathrm{Hu}^{\mathrm{a}}$, Tao Lin ${ }^{\mathrm{a}}$, Fengyuan Che ${ }^{\mathrm{d}}$, Qi Wan ${ }^{\mathrm{a}, \mathrm{e}}$

${ }^{a}$ Institute of Neuroregeneration $\&$ Neurorehabilitation, Department of Pathophysiology, Qingdao University, 308 Ningxia Street, Qingdao 266071, China

${ }^{b}$ Department of Physiology, School of Medicine, Wuhan University, 185 Donghu Street, Wuhan 430071, China

${ }^{c}$ Department of Rehabilitation, Affiliated Hospital of Qingdao University, Qingdao, 266000, China.

${ }^{d}$ Central Laboratory, Department of Neurology, Linyi People's Hospital, Qingdao University, 27 East Jiefang Road, Linyi, Shandong, China

${ }^{e}$ Qingdao Gui-Hong Intelligent Medical Technology Co. Ltd, 7 Fenglong Road, Qingdao High-tech Industrial Development District, Qingdao, China

Correspondence:

Qi Wan, Institute of Neuroregeneration \& Neurorehabilitation, Department of Pathophysiology, Qingdao University, 308 Ningxia Street, Qingdao 266071, China. Email: qiwan1@hotmail.com

Fengyuan Che, Central Laboratory, Department of Neurology, Linyi People's Hospital, Qingdao University, 27 East Jiefang Road, Linyi, Shandong, China

Email: che1971@126.com 


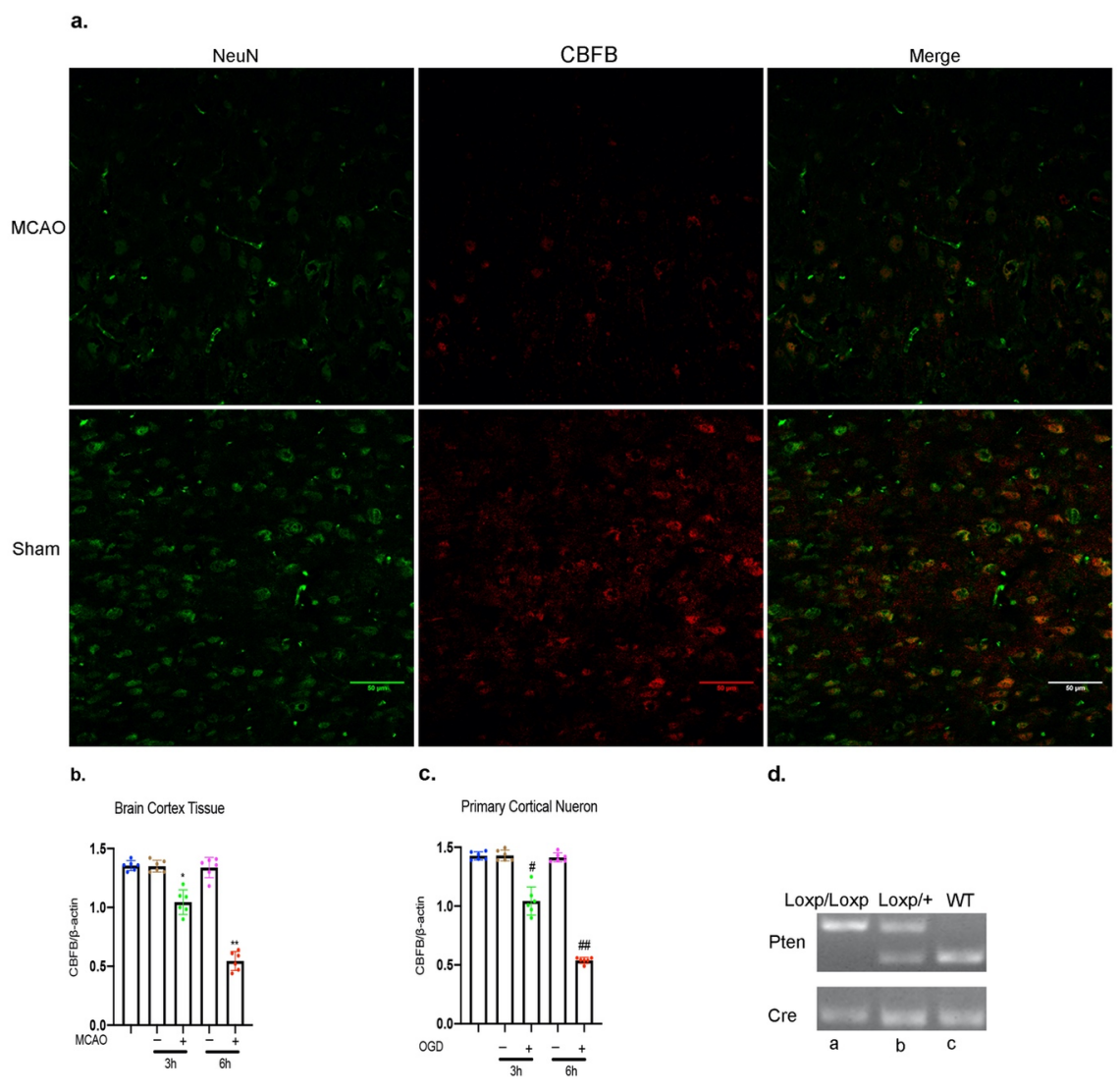

\section{Supplementary Fig. 1 CBFB is decreased in neuron after ischemic injury}

1. Immunofluorescence of CBFB in cerebral cortex. Neuron (NeuN, green), CBFB (red)

2. Western blotting analysis of CBFB in cortex tissue after $I / R$ injury. Peri-infarct cortex tissue was prepared for western blotting analysis at $3 \mathrm{~h}, 6 \mathrm{~h}$ after $60 \mathrm{~min}$ occlusion $\left(\mathrm{n}=6\right.$ in each group, ${ }^{*} \mathrm{p}<0.05$ versus $3 \mathrm{~h}$ sham, ${ }^{* *} \mathrm{p}<0.05$ versus $6 \mathrm{~h}$ sham, one-way ANOVA test).

3. Western blotting analysis of CBFB in primary neuron after OGD insult. Primary cortical neuron was prepared for western blotting analysis at $3 \mathrm{~h}, 6 \mathrm{~h}$ after $60 \mathrm{~min}$ OGD insult ( $\mathrm{n}=6$ in each group, ${ }^{\#} \mathrm{p}<$ 0.05 versus $3 \mathrm{~h}$ sham, ${ }^{\# \#} \mathrm{p}<0.05$ versus $6 \mathrm{~h}$ sham, one-way ANOVA test)

4. Genotyping of Pten conditional- knockout mouse compared to wild-type, Pten -/-(a), Pten +/- (b), $\mathrm{WT}(\mathrm{c})$ 
a.

Primary Cortical Neuron

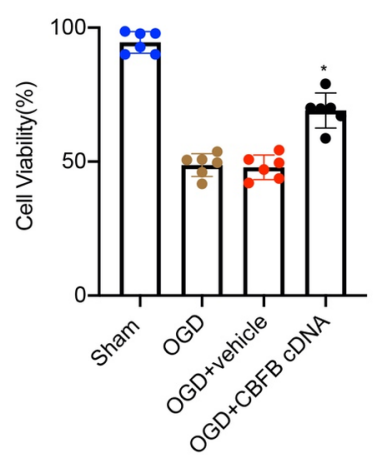

c. Primary Cortical Neuron

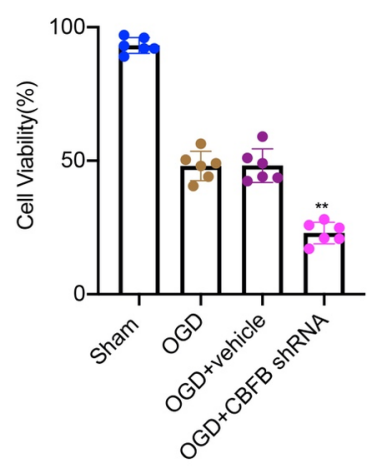

b.

Primary Cortical Neuron

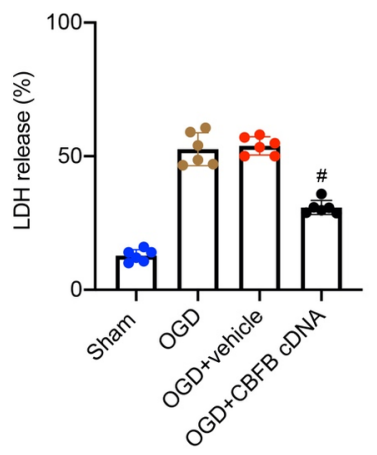

d.

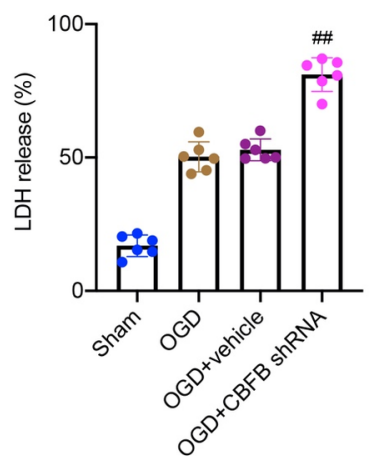

\section{Supplementary Fig. 2 Downregulation of CBFB mediates neuronal death after OGD insult}

1. Cell viability test shows overexpression of CBFB reduces neuronal death after OGD insult. CBFB cDNA is transfected into neuron $72 \mathrm{~h}$ prior to OGD insult ( $\mathrm{n}=6$ in each group, ${ }^{*} \mathrm{p}<0.05$ versus OGD + Vehicle, one-way ANOVA test).

2. LDH release test shows overexpression of CBFB reduces neuronal death after OGD insult. CBFB cDNA was transfected into neuron $72 \mathrm{~h}$ prior to OGD insult $\left(\mathrm{n}=6\right.$ in each group, ${ }^{*} \mathrm{p}<0.05$ versus OGD + Vehicle, one-way ANOVA test).

3. Cell viability test shows knockdown of CBFB promotes neuronal death after OGD insult. CBFB shRNA was transfected into neuron $72 \mathrm{~h}$ prior to OGD insult $\left(\mathrm{n}=6\right.$ in each group, ${ }^{* *} \mathrm{p}<0.05$ versus OGD + Vehicle, one-way ANOVA test).

4. LDH release test shows knockdown of CBFB promotes neuronal death after OGD insult. CBFB shRNA was transfected into neuron $72 \mathrm{~h}$ prior to OGD insult ( $\mathrm{n}=6$ in each group, ${ }^{\# \#} \mathrm{p}<0.05$ versus OGD + Vehicle, one-way ANOVA test). 
a.

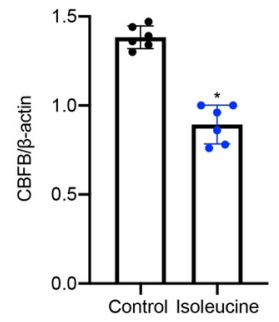

b.

Primary Cortical Neuron

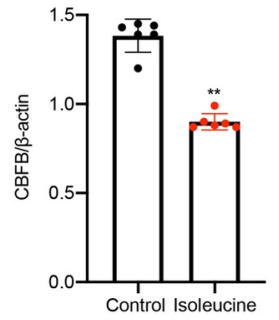

\section{Supplementary Fig. 3 Isoleucine decreases the expression of CBFB in rat neurons}

Isoleucine decreases the expression of CBFB in cortex. Isoleucine $(10 \mathrm{mg} / \mathrm{kg})$ is intraperitoneally injected $6 \mathrm{~h}$ before the rat cortex is prepared for western blotting analysis $\left(n=6\right.$ in each group, ${ }^{*} \mathrm{p}<0.05$ versus control, one-way ANOVA test).

Isoleucine decreases the expression of $\mathrm{CBFB}$ in primary neuron. Isoleucine is supplemented to neuron $6 \mathrm{~h}$ before primary neuron culture is prepared for western blotting analysis $\left(\mathrm{n}=6\right.$ in each group, ${ }^{* *} \mathrm{p}<0.05$ versus control, one-way ANOVA test). 
Fig. 1

a.

CSF

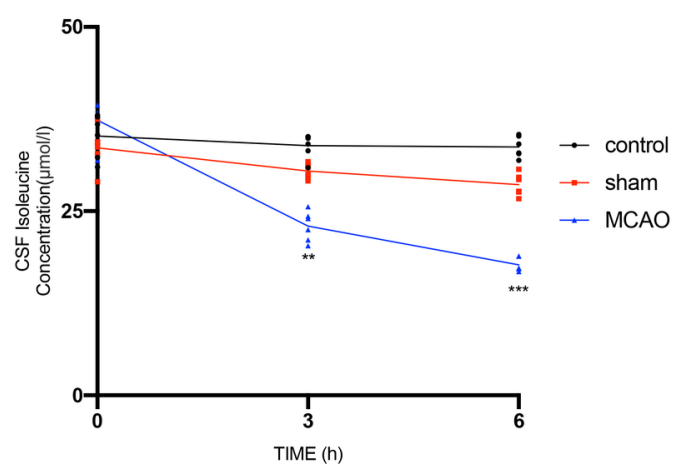

b.

Primary Cortical Neuron

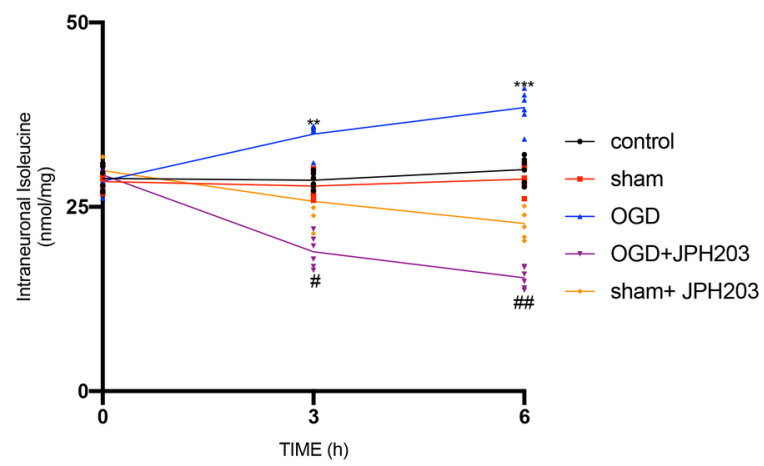




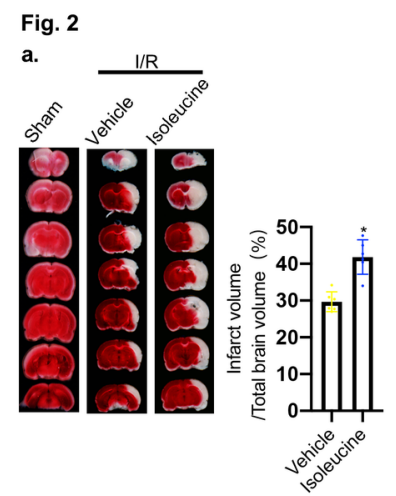

b.

c.
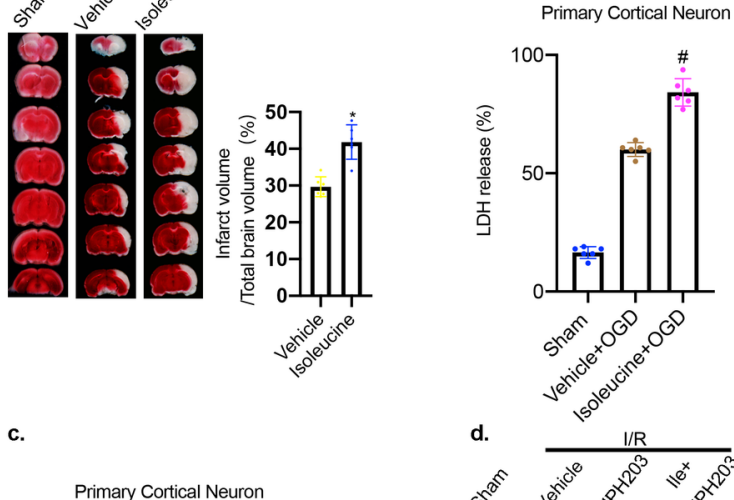

d.
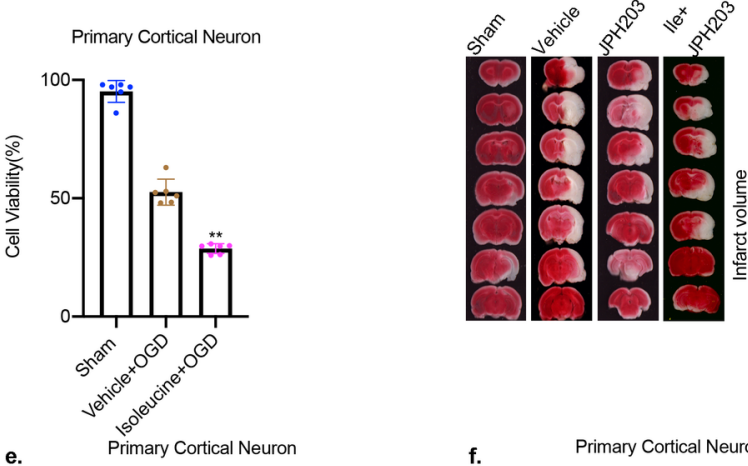

]

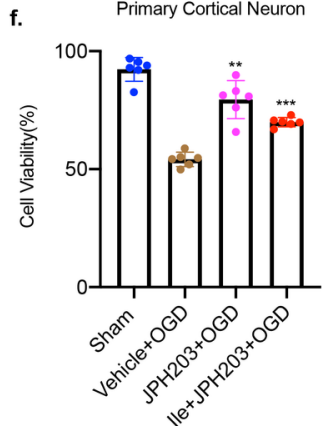


Fig. 3
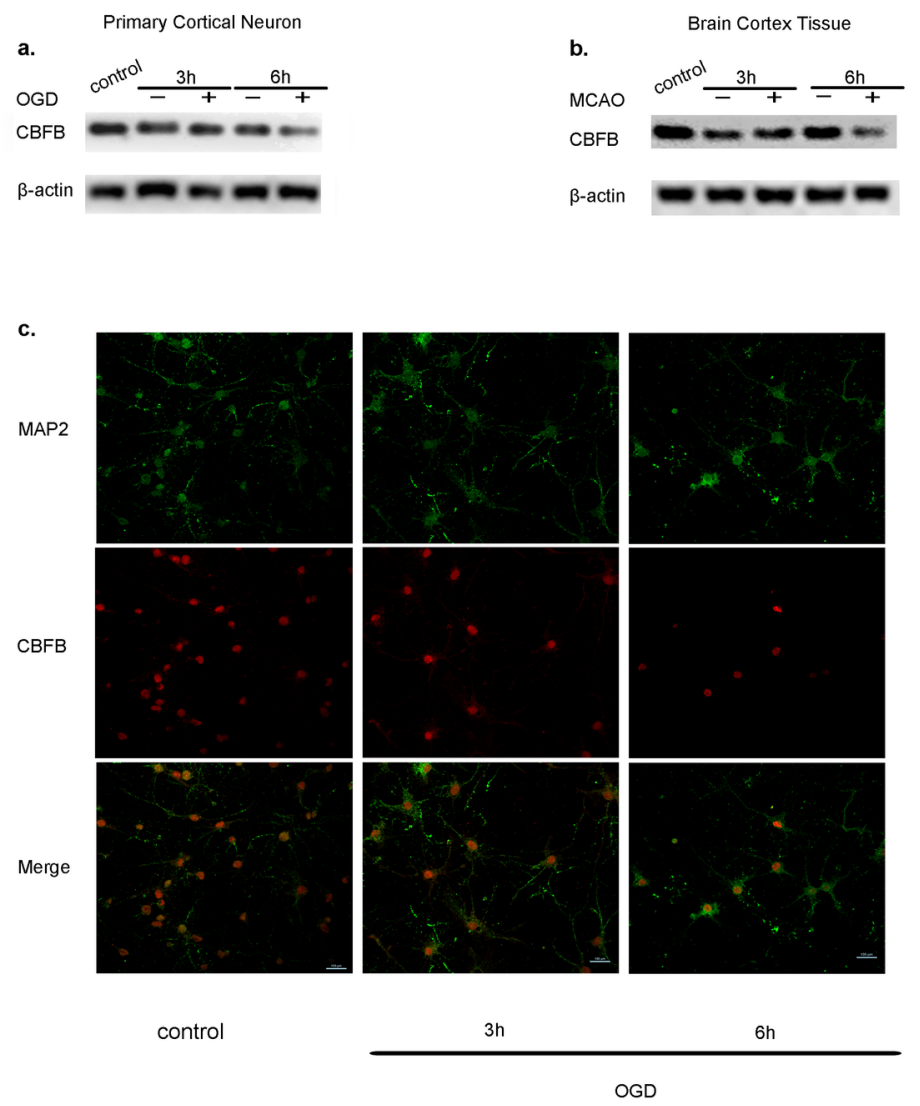
Fig. 4

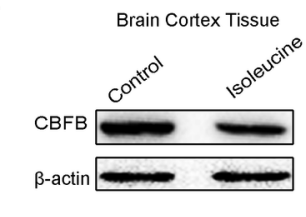

c.
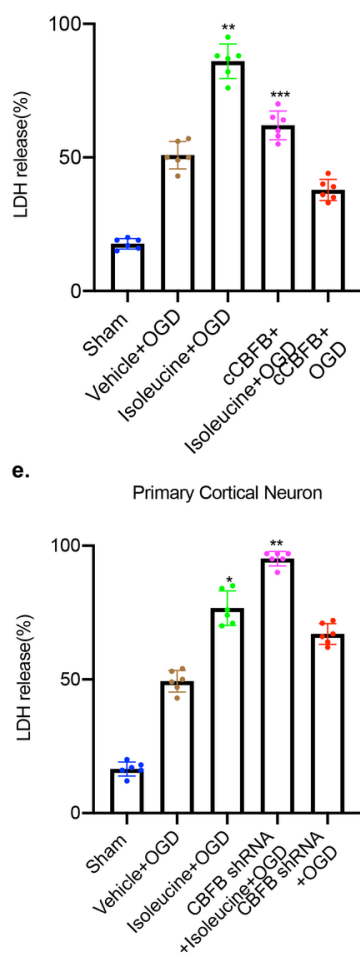

b.

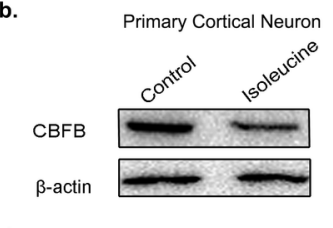

d.

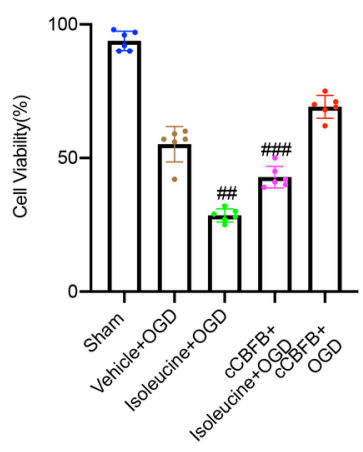

f.
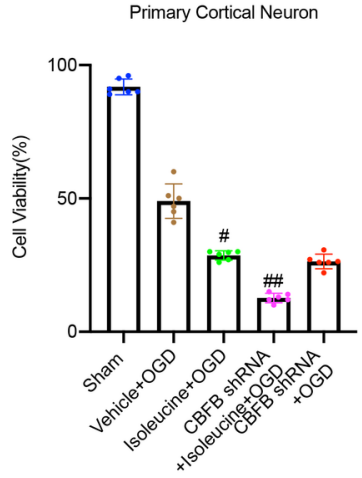
Fig. 5
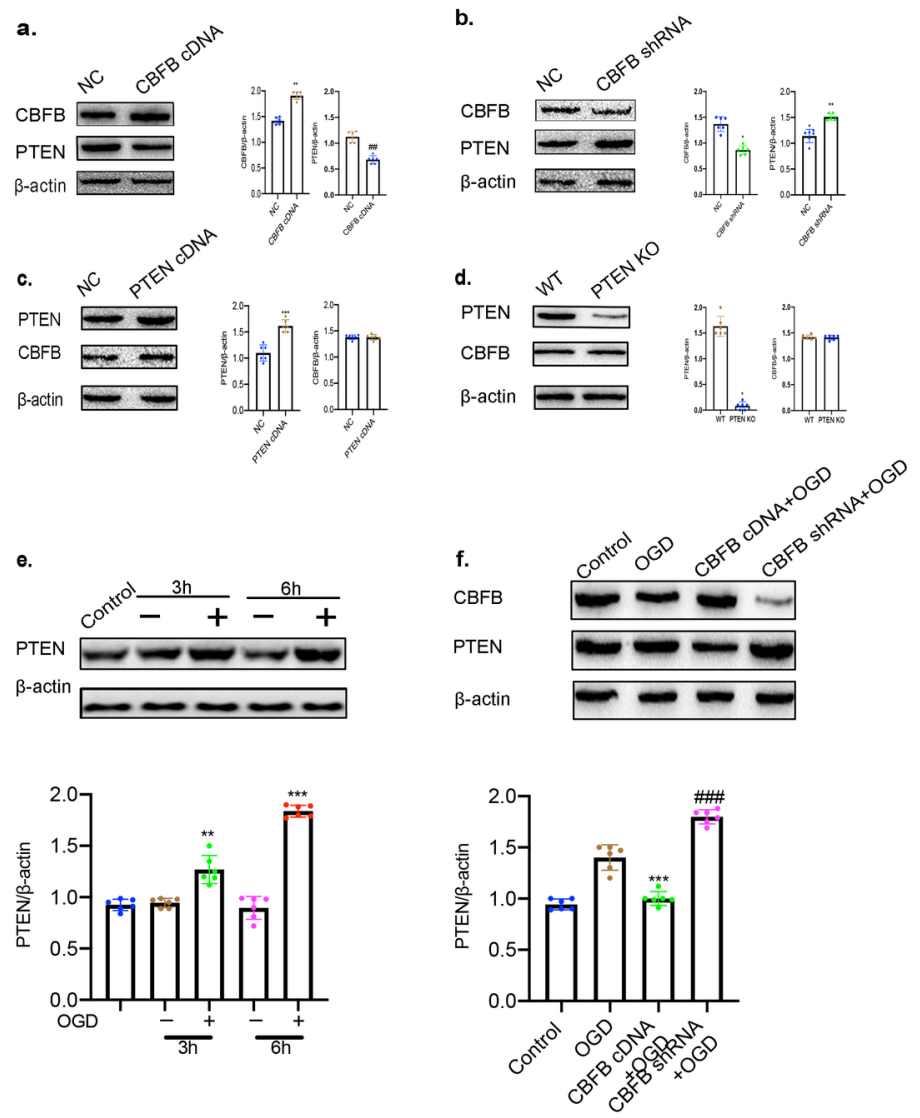

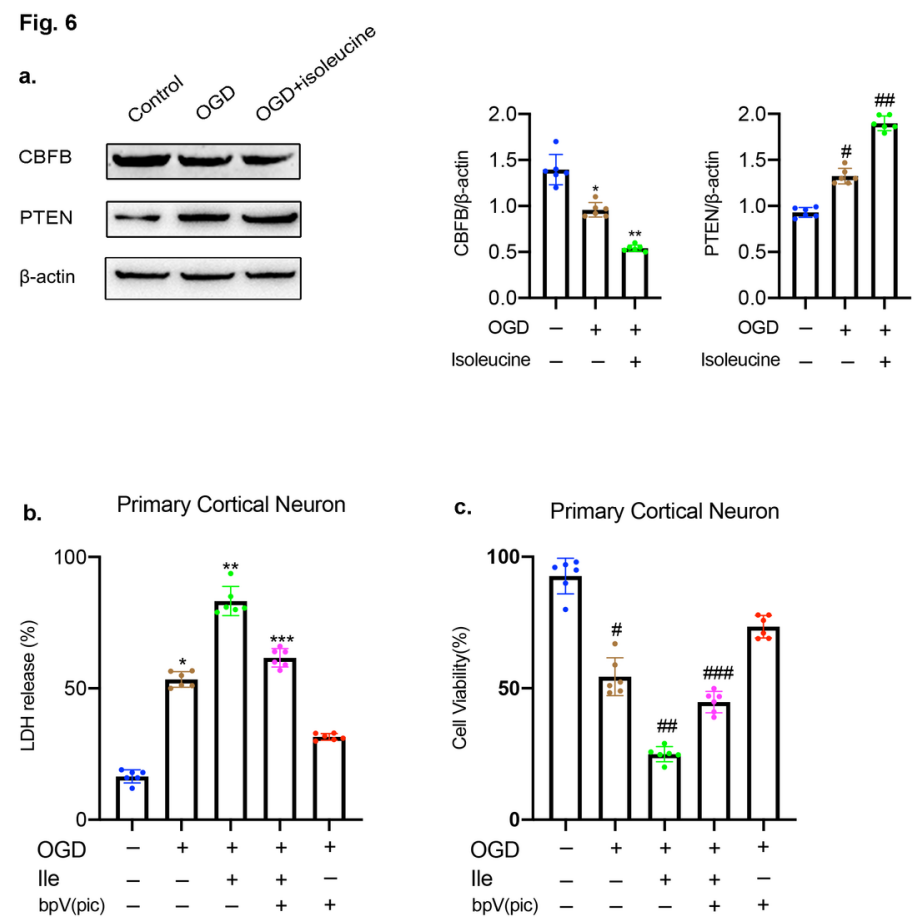
Fig. 7

a.
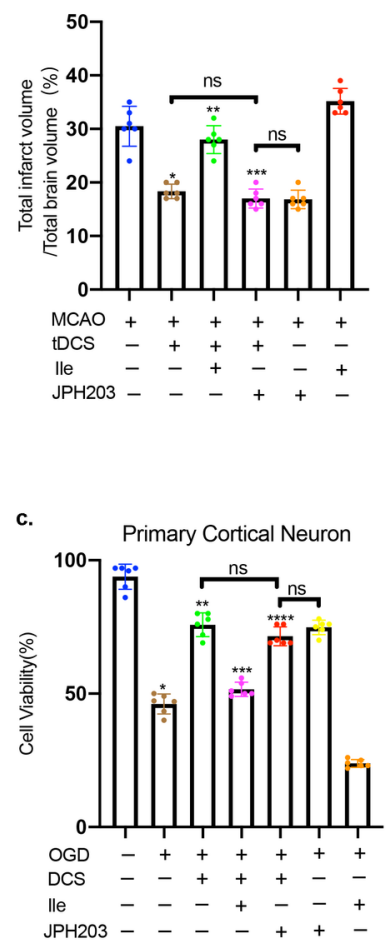

b.
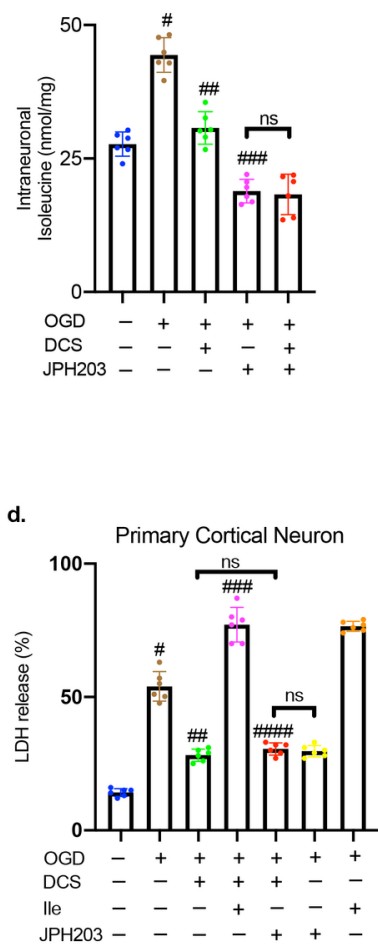